\title{
Ein Bronzedolch des Simbar-Šipak von Babylon (1025-1008) Überlegungen zu Waffenweihungen im Vorderen Orient
}

\author{
von Karen Radner und Stephan Kroll - München
}

Ein Bronzedolch, der derzeit in der Schausammlung des Archäologischen Museums von Tabriz (Iran) ausgestellt ist, ${ }^{1}$ gehört zu einer prominenten Gruppe innerhalb der sogenannten „Luristanbronzen“, nämlich der der beschrifteten Randgriffdolche. Diese Waffen sind mit Inschriften verschiedener Herrscher von Babylon der letzten beiden Jahrhunderte des II. Jahrtausend v. Chr. versehen, gelegentlich auch mit Inschriften von nicht-königlichen Personen, darunter ein Schreiber und ein ša rēšs sarri. ${ }^{2}$

Die bisher bekannten Königsinschriften auf diesen Dolchen stammten von Herrschern der [1] Kassitendynastie, der [2] II. Dynastie von Isin und der [3] Dynastie von Bazi: [1] Adad-šuma-ușur (1211-1182); [2] Ninurta-nādin-šumi (1131-1126), Nabû-kudurrī-uṣur (Nebukadnedzar) I. (1125-1104), Enlil-nādin-apli (1103-1100), Marduk-nādin-ahḩ̄ē (10991082), Marduk-šāpik-zēri (1081-1069), Adad-apla-iddina (1068-1047); [3] Eulmaš-šākin-šumi (1004-988). ${ }^{3}$

1 Michael Roaf fertigte im April 2002 im Rahmen einer Exkursion der Universität München, an der auch Karen Radner teilnahm, einige Aufnahmen des Dolches an, nach denen Cornelie Wolff die Zeichnung erstellte. Die Kopie des Keilschrifttextes stammt von Karen Radner und beruht auf Detailphotographien von Herrn Christian Schattauer (München) aus dem Sommer 2004; den Kontakt stellte Stephan Kroll her, der über Herrn Bahram Adjerloo (Teheran) Informationen zur Herkunft und zu den Dimensionen des Dolchs in Erfahrung bringen konnte. Die Autoren sind allen genannten Personen für ihre Hilfe zu großem Dank verpflichtet, außerdem Ursula CalmeyerSeidl und Frans van Koppen für wertvolle Literaturhinweise.

2 Zusammengestellt bei J. A. Brinkman, A Political History of Post-Kassite Babylonia 1158-722 B.C. AnOr. 43 (Rom 1968) 11; weiteres S. M. Maul, Ein beschriftetes Schwert aus der späten mittelbabylonischen Zeit, NABU 1995 Nr. 74.

3 Zusammengestellt bei P. Calmeyer, Datierbare Bronzen aus Luristan und Kirmanshah. UAVA 5 (Berlin 1969) 59-64 (der Nachtrag auf S. 64 ist zu beachten!) und P. R. S. Moorey, Catalogue of the Ancient Persian Bronzes in the Ashmolean Museum (Oxford 1971) 29-34; vgl. auch die Tabelle bei B. Sass, Inscribed Babylonian Arrowheads of the Turn of the Second Millennium and Their Phoenician Counterparts, UF 21 (1989) 353. Inschriften: Adad-šuma-ușur $=\mathrm{G}$. Dossin, Bronzes inscrits du Luristan de la collection Foroughi, Iranica Antiqua 2 (1962) 151 Nr. 1-2, f. XIIIf. (vgl. J. A. Brinkman, Materials and Studies for Kassite History 1 [Chicago 1976] 90f. C.2.2.-3.); Ninurta-nādin-šumi = 
Mit dem hier vorgestellten Stück ist nun erstmals auch ein Herrscher der II. Dynastie des Meerlandes nachzuweisen, der einen bronzenen Randgriffdolch mit seiner Inschrift versehen ließ: Die Waffe trägt auf der Vorderseite eine zweizeilige Keilinschrift in akkadischer Sprache, die den babylonischen König Simbar-Šipak ${ }^{4}$ (1025-1008) nennt, den Begründer der II. Meerlanddynastie. 5 Damit wird erst die dritte Inschrift bekannt, die auf diesen Herrscher zurückgeht. Sie lautet:
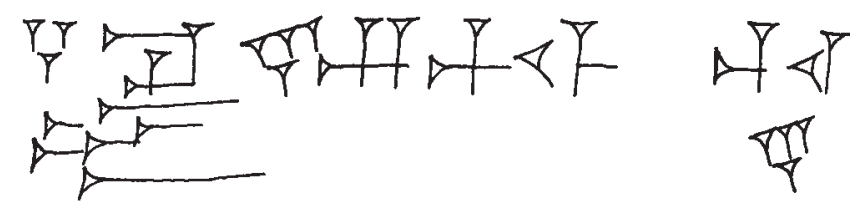

1 šá Si-im-bar-Ši-pak

2 LUGAL ŠÁR

„(Besitz) des Simbar-Šipak, Königs der Gesamtheit.“

Die Inschrift entspricht jenem Formular der Dolchaufschriften, das auch für die Könige Marduk-šāpik-zēri, Adad-apla-iddina und Eulmaššākin-šumi belegt ist und damit typisch für die jüngeren Vertreter der Denkmälergruppe ist (Typ D in der folgenden Tabelle): Die Titulatur ist auf „König der Gesamtheit“ beschränkt und verzichtet auf die Titel „König von Babylon“ und „König von Sumer und Akkad“, wie sie für den bei den älteren Stücken verwendeten Typ C belegt sind, sowie auf die Angabe des Vaters (Typ B). Im Gegensatz zu einem frühen Beispiel von Adad-šuma-ușur (Typ A) wird die Inschrift jedoch mit dem Eigentumsvermerk $\check{s} a$ eingeleitet.

Bei den beiden anderen bekannten Inschriften des Simbar-Šipak handelt es sich einerseits um die lange Inschrift auf dem Thron des Enlil aus dessen Heiligtum in Nippur, die allerdings nur in Form von zwei späteren Abschriften überliefert ist, ${ }^{6}$ andererseits um die Inschrift auf einer bronzenen Pfeilspitze aus einem unbekannten Herkunftsort in Iran; letztere lautet: ${ }^{1}$ šá Si-im-bar-Ši-pak ${ }^{2}$ DuMu ${ }^{1}$ Eri-ba-d30 ,(Besitz) des

RIMB 2 B.2.3.1, Nabû-kudurrī-ușur I. = RIMB 2 B.2.4.3, Enlil-nādin-apli = RIMB 2 B.2.5.1, Marduk-nādin-ahḩe = RIMB 2 B.2.6.4, Marduk-šāpik-zēri = RIMB 2 B.2.7.3, Adad-apla-iddina = RIMB 2 B.2.8.12; Eulmaš-šākin-šumi = RIMB 2 B.4.1.2.

${ }^{4} \mathrm{Ob}$ das theonyme Element des Namens als Šipak oder Šihu gelesen werden soll, ist nicht geklärt.

$5 \mathrm{Zu}$ diesem Herrscher s. Brinkman, Political History (Anm. 2) 45 f., 150-155, 340 und G. Frame, Rulers of Babylonia from the Second Dynasty of Isin to the End of Assyrian Domination (1157-612 BC). RIMB 2 (Toronto 1995) 71.

${ }^{6}$ RIMB 2 B.3.1.1. 


\begin{tabular}{|c|c|c|}
\hline \multicolumn{3}{|l|}{ Kassiten } \\
\hline $\begin{array}{r}\text { Adad-šuma-ușur } \\
(1211-1182)\end{array}$ & $\begin{array}{l}\text { (Vs.) }{ }^{\mathrm{I}} \mathrm{IM}-\mathrm{MU}-\check{\mathrm{S} E S ̌ ~ L U G A L ~ K I S ̌ ~} \\
\text { (Vs.) } \check{s} a \mathrm{~d} \mathrm{IM}-\mathrm{MU}-\check{\mathrm{S} E S ̌ S} \text { LUGAL ŠÁR } \\
\text { (Rs.) DUMU Kaš-til-ia-šu LUGAL KÁ.DINGIR.RA }{ }^{\mathrm{ki}}\end{array}$ & $\begin{array}{l}\text { Typ A } \\
\text { Тур B }\end{array}$ \\
\hline \multicolumn{3}{|l|}{$I \sin I I$} \\
\hline $\begin{array}{r}\text { Ninurta-nādin-šumi } \\
(1131-1126) \\
{[2 \times]}\end{array}$ & $\begin{array}{l}\text { (Vs.) } \check{s} a \mathrm{~d} N i n-u r t a-S U M-M U \text { LUGAL ŠÁR } \\
\text { (Rs.) LUGAL KÁ.DINGIR.RA }{ }^{\text {ki }} \text { LUGAL KI.EN } 6 \text {.GI URI }{ }^{\mathrm{ki}}\end{array}$ & Typ C \\
\hline $\begin{array}{r}\text { Nebukadnedzar I. } \\
(1125-1104) \\
{[4 \times]}\end{array}$ & 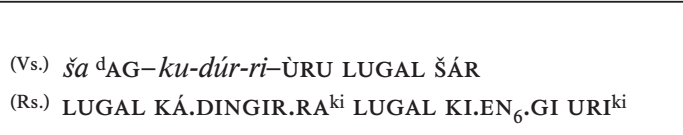 & Typ C \\
\hline $\begin{array}{r}\text { Enlil-nādin-apli } \\
(1103-1100)\end{array}$ & 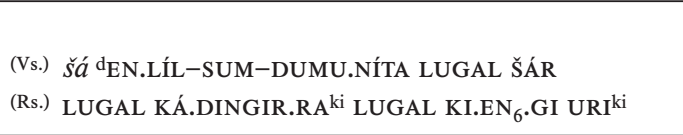 & Typ C \\
\hline $\begin{array}{r}\text { Marduk-nādin-ahḩ̄e } \\
(1099-1082) \\
{[4 \times]}\end{array}$ & $\begin{array}{l}\text { (Vs.) } \breve{s a ́ ~ d A M A R . U T U-S U M-S ̌ E S ̌ ~ L U G A L ~ S ̌ A ́ R ~} \\
\text { (Rs.) DUMU d Nin-urta-SUM-MU LUGAL KÁ.DINGIR.RA }{ }^{\mathrm{ki}}\end{array}$ & Typ B \\
\hline $\begin{array}{r}\text { Marduk-šāpik-zēri } \\
(1081-1069)\end{array}$ & (Vs.) $\check{s} a$ dAMAR.UTU- $\breve{a} a-p i ́-i k$-NUMUN (Rs.) LUGAL ŠÁR & Typ D \\
\hline $\begin{array}{r}\text { Adad-apla-iddina } \\
(1068-1047)\end{array}$ & (Vs.) $\check{s} a$ dIM-DUMU.NÍTA-SUM ${ }^{n a}$ LUGAL ŠÁR & Typ D \\
\hline \multicolumn{3}{|l|}{ Meerland II } \\
\hline $\begin{array}{r}\text { Simbar-Šipak } \\
(1025-1008)\end{array}$ & (Vs.) šá $S i$-im-bar-Ši-pak LUGAL ŠÁR & Typ D \\
\hline \multicolumn{3}{|l|}{ Bazi } \\
\hline $\begin{array}{r}\text { Eulmaš-šākin-šumi } \\
(1004-988)\end{array}$ & (Vs.) šá É.UL.MAŠ-GAR-MU LUGAL [ŠÁR] & Typ D \\
\hline
\end{tabular}

Simbar-Šipak, des Sohnes des Eriba-Sîn."7 Außerdem ist ein Kudurru aus der Regierungszeit dieses Herrschers bekannt. ${ }^{8}$

Beim vorliegenden Inschriftenträger handelt sich um einen langen Bronzedolch mit stark geschwungenem Griff. Nach Auskunft von Bahram Adjerloo 9 beträgt die Länge des Dolchs $75 \mathrm{~cm}$, die Breite der Klinge $5 \mathrm{~cm}$. Die nächste Parallele ist ein Stück mit einer Inschrift des Adad-apla-iddina

\footnotetext{
7 RIMB 2 B.3.1.2.

8 BM 90937 = BBSt 27.

9 Email vom 20. Juni 2005.
} 


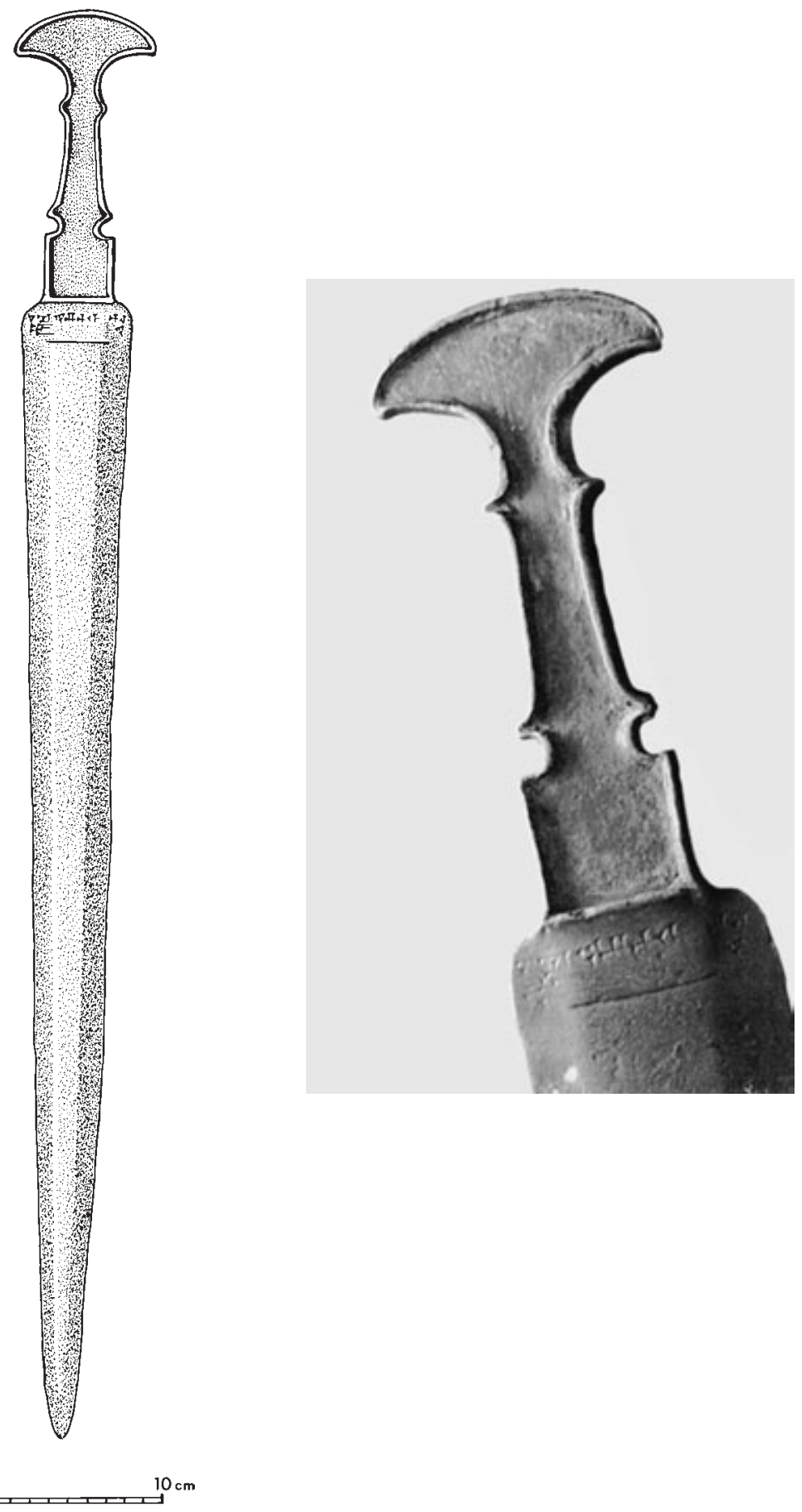


von Babylon (1068-1047), ${ }^{10}$ wenn auch der Griff unseres Dolches dreifach, nicht zweifach eingezogen ist und seine Klinge deutlich länger ist. Die Inschrift ist bei unserem Stück, wie bei den meisten vergleichbaren Exemplaren auch, direkt unterhalb des Hefts und parallel zu diesem auf der Klinge angebracht. Wesentlich kürzer ist dagegen ein Dolch mit Inschrift des Eulmaš-šākin-šumi (1004-988), ${ }^{11}$ dessen zweizeilige Inschrift sich auf der Klinge selbst befindet und nicht dicht unterhalb des Griffes.

Nach den verfügbaren Informationen ${ }^{12}$ stammt unser Dolch aus der Stadt Marāgheh in der modernen iranischen Provinz Ost-Azerbaijan, wo ihn ein Bauer in einem Bach gefunden haben soll. Marāgheh liegt $22 \mathrm{~km}$ östlich des Urmia-Sees auf einer Höhe von 1619 m im Süden des Kūh-e Sahand-Massivs (3700 m). Bisherige Herkunftsangaben für derartige Dolche nennen fast immer Kermanshah oder Luristan („Luristanbronzen“), 13 nie jedoch Kurdistan oder Azerbaijan. ${ }^{14}$ Marlik weist jedoch in seinem Fundkontext zwei entfernt vergleichbare Dolche auf,15 und ähnliche Stücke könnten eventuell auch in Hasanlu IVB im Kontext des 10.-9. Jh. v.C. vorkommen: Bisher hat M. de Schauensee allerdings nur skizzenhaft Griffe von Randgriffdolchen mit mehreren Einzügen bzw. Ausstülpungen vorgestellt. ${ }^{16}$ In Gilan am Kaspischen Meer wurden solche Formen dagegen nicht gefunden, ${ }^{17}$ und weiter nördlich in Transkaukasien sind derartige Dolche ebenfalls nicht belegt. ${ }^{18}$ Auch die Grabungen in den eisenzeitlichen Gräbern von Dinkha Tepe, südwestlich des Urmiasees, lieferten kein entsprechendes Material. ${ }^{19}$ Obwohl die Stadt Marāgheh im Ruf eines

10 Calmeyer, Datierbare Bronzen (Anm. 3) 61 mit Abb. 61: Gruppe 31 Q.

11 W. G. Lambert, Three Inscribed Luristan Bronzes, AfO 22 (1968/69) 9-11 Abb. 2. Der Dolch ist Teil der Sammlung Ligabue in Venedig; s. auch: F. M. Fales, Prima dell'alfabeto (Venedig 1989) 245.

12 Die Information der Vitrinenbeischrift bestätigte Bahram Adjerloo, auf den alle weiteren Details zurückgehen (Email vom 23. November 2004).

13 Vgl. B. Overlaet, The Early Iron Age in the Pusht-i Kuh. Luristan Excavation Documents IV. Acta Iranica 40 (Leuven 2003) 163-166.

14 Calmeyer, Datierbare Bronzen (Anm. 3) $62 \mathrm{f}$.

15 E. O. Negahban, Weapons from Marlik. AMI Erg. 16 (Berlin 1995) 48, Tf. V Abb. 52-53.

16 M. de Schauensee, Northwest Iran as a Bronze-Working Centre: The View from Hasanlu, in: J. E. Curtis (Hg.), Bronzeworking Centres (Anm. 17) 45-62 Tf. 51 c.d.g.

17 E. Haerinck, The Iron Age in Guilan. Proposal for a Chronology, in: J. E. Curtis (Hg.), Bronzeworking Centres of Western Asia, ca. 1000-539 B.C. (London/New York 1988) 63-78 Tf. 61-72.

18 Vgl. A. Schachner, Von Bronze zu Eisen: Die Entwicklung der Metallurgie während des 2. Jahrtausends v. Chr. im östlichen Transkaukasus, in: Ü. Yalçın (Hg.), Anatolian Metal III. Der Anschnitt Beih. 18 (Bochum 2004) 175-190 Abb. 4-8.

19 O. W. Muscarella, The Iron Age at Dinkha Tepe, Iran, The Metropolitan Museum Journal 9 (1974) 35-90. 
Umschlagplatzes für Antiquitäten steht, ist sie wegen der Stücke aus Marlik und Hasanlu als Fundort unseres Dolches nicht völlig auszuschließen; insbesondere ist zu bedenken, daß es in Marāgheh selbst für den fraglichen Zeitraum keine kontrollierten Grabungsbefunde gibt.

Nachdem keine durch archäologische Ausgrabungen gesicherten Informationen zum Fundort vorliegen, stellt sich bei unserem Stück genauso wie bei den übrigen beschrifteten Waffen (Dolche, Pfeilspitzen und Beile) die Frage, inwieweit Aussagen zum Herkunftsort und, damit eng verbunden, zum Verwendungszweck gemacht werden können. Diese Diskussion ist nicht neu und wurde wiederholt von Archäologen wie auch Philologen, die insbesondere eine Erklärung für die Herkunft aus dem iranischen Hochland finden wollten, auf der Basis der Denkmäler geführt. Die Stücke wurden deshalb einerseits als Ehrengeschenke für iranische Söldner, die babylonischen Königen treu gedient hätten, gedeutet, ${ }^{20}$ aber auch als Votivgaben, die von Invasoren aus babylonischen Tempeln nach Iran verschleppt worden wären. ${ }^{21}$ Für die Meinung, daß es sich bei vielen, wenn nicht gar allen Stücken um Weihgaben handle, wird allgemein auf jene beschrifteten Waffen verwiesen, die Weihinschriften tragen: Die Inschriften zweier Dolche mit nicht-königlichen Inschriften weisen ihre Trägerobjekte als Weihgaben an Marduk ${ }^{22}$ bzw. den „Gott meiner Stadt“ ${ }^{23}$

20 Zuletzt wurde diese Meinung von Sass, UF 21 (1989) 354 vertreten. Daß Schwerter und Dolche mit Namensbeischrift eines Herrschers als Ehrengeschenke an seine Günstlinge verliehen werden konnten, steht außer Frage: So trifft Assurbanipal von Assyrien (668-ca. 630) in Bezug auf seinen Vasallen Necho von Sais in Ägypten folgende Aussage: „Ein mit Gold eingelegtes Gürtelschwert beschriftete ich mit meinem Namenszug und gab es ihm“ (R. Borger, Beiträge zum Inschriftenwerk Assurbanipals [Wiesbaden 1996] 23, 214: Prisma A II 12-13 // C III 13-14: Gír.AN.BAR // GÍR šib-bi šá ih-zu-šú KÙ.GI ni-bit MU-ia ina UGU-hi áš-țur-ma ad-din-šú // a-din-šú).

${ }^{21}$ Zusammenfassung der Diskussion (mit älterer Literatur) bei Brinkman, Political History (Anm. 2) $9 \mathrm{f}$. und Calmeyer, Datierbare Bronzen (Anm. 3) $170 \mathrm{f}$.

${ }^{22}$ W. Nagel, Die Königsdolche der Zweiten Dynastie von Isin, AfO 19 (1959-60) 96: šá ${ }^{1}$ Eri$b a-\mathrm{d}$ NUSKU tup-šar-ri šá dAMAR.UTU ÁG-mu la i-ta-bal-šu ,(Besitz/Weihung) des Schreibers Erïba-Nusku. Wer Marduk liebt, soll ihn (d.h., den Dolch) nicht wegnehmen!“

23 Dossin, Iranica Antiqua 2 (1962) 153 Nr. 7, Tf. XIV: $a$-na DINGIR URU-ia EN GAL- $i$ qar-di șe-ri ${ }^{1 \mathrm{~d}}$ AMAR.UTU-ŠEŠ $a$-na TI.LA ZI.MEŠ-šu i-qí-iš „Dem, Gott meiner Stadt', dem großen, heldenhaften und erhabenen Herrn, hat Marduk-nāșir (diesen Dolch) für sein Leben geweiht.“ Brinkman, Political History (Anm. 2) 9 Anm. 35, 362 liest den Göt-

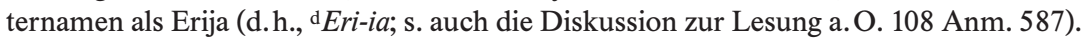
Nachdem aber die Ansprache des örtlichen Hauptgottes als ,Gott meiner Stadt' gut belegt ist (für Belege s. CAD I/J 93; außerdem AbB 5 239: 32, AbB 8 99: 21, AbB 10 178: 4, 15, AbB 12 186: 12, etc.), ziehe ich die lectio facilior vor, wie sie schon Dossin a. O. verwendet hat (vgl. W. G. Lambert, Studies in Marduk, BSOAS 47 [1984] 9: „There is no god Erīya, only il-āli-ia.“). Der Wechsel zwischen der ersten und der drit- 
aus, ebenso wie zwei Beile mit Inschriften Nebukadnedzars I. (1125-1104) durch das die Inschrift einleitende Gebet an Marduk als Weihungen an diesen Gott nach einer siegreichen Schlacht gekennzeichnet sind: „Du vermagst, Marduk, den, der ständig (zu dir) betet, zu retten, den, der deine heiligen Stätten immer wieder aufsucht, über die Feinde zu stellen. Dein Hauch wehte zu mir, da konnte ich meine Feinde niederwerfen. Mach meine Waffen stark, damit ich meine Gegner zu Fall bringen kann! (Besitz/Weihung) des Nebukadnedzar, des Königs der Gesamtheit."24

Wir wollen die Diskussion nicht neu aufrollen, sondern zusätzliche Argumente dafür einführen, (1) daß es sich bei den Objekten um Weihgaben handelt und (2) daß die Annahme, daß diese einem in Babylonien gelegenen Heiligtum entstammen, nicht zwingend notwendig ist.

Für die altbabylonische Zeit läßt sich belegen, daß dem Gott Nergal in seinem Heiligtum in der Stadt Hubšalum Schwerter geweiht wurden und daß damit siegreiche Feldherren ihre entsprechenden Gelübde, die die Waffenhilfe des Kriegsgottes sichern sollten, nach der erfolgreich geschlagenen Schlacht einlösten. Dominique Charpin ${ }^{25}$ konnte diese Praxis nachweisen, indem er eine entsprechende Passage in einem Brief aus der Königskorrespondenz Zimrī-Līms von Mari (1677-166426) mit jenem mit einer Weihinschrift versehenen Bronzeschwert in Beziehung setzte, das H. G. Güterbock im Jahr 1965 publiziert hatte. ${ }^{27}$ Entlang der Klinge dieses $109 \mathrm{~cm}$ langen Schwertes mit einem von zwei Löwen dekorierten Griff ist eine akkadische Inschrift in einer Zeile angebracht: „Dem Herrn von Hubšalum, seinem Herrn, hat Luluanum, der Sohn des Azizum, für sein Leben und das Leben seiner Söhne ein Schwert von zwölf Minen (Gewicht) geweiht (wört. eintreten lassen).“28 Wer dieser Luluanum war, wissen wir nicht, doch steht jene Waffenweihung, die für Zimrī-Lìm überliefert ist, ganz in der Tradition seines Schwertes. Der König von Mari wurde in einem Brief des Šamaš-Heiligtums von Sippar aufgefor-

ten Person ist auch sonst bei Verweis auf die persönliche Beziehung zu einer Gottheit im Inschriftenmaterial zu belegen (z.B. RIME 4.2.13a.2: 45).

24 RIMB 2, B.2.4.4. Die Übersetzung folgt W. Sommerfeld, Der Aufstieg Marduks. AOAT 213 (Kevelaer/Neukirchen-Vluyn 1982) 184.

25 D. Charpin, L'épée offerte au dieu Nergal de Hubšalum, NABU 1989/76.

26 Nach der „ultrakurzen Chronologie“ von H. Gasche u.a., Dating the Fall of Babylon. A Reappraisal of Second-Millennium Chronology. MHEM 4 (Ghent/Chicago 1998).

${ }^{27}$ H. G. Güterbock, A Votive Sword with Old Assyrian Inscription, in: H. G. Güterbock/T. Jacobsen (Hg.), Studies in Honor of Benno Landsberger. AS 16 (Chicago 1965) $197 \mathrm{f} .$, Tf. xiii-xv.

28 a-na be-lim ša Hu-ub-ša-lim be-lí-šu Lu-lu-a-num DUMU A-zi-zi-im a-na ba-lá-ți-šu ú ba-lá-at ma-ri-šu GÍR ša 12 MA.NA ú-šé-ri-ib. Für die Lesung des Ortsnamen in dieser Inschrift s. Charpin, NABU 1987/76. 
dert, sein Gelübde an Nergal von Hubšalum nach dem Sieg über die Truppen Išme-Dagāns von Ekallātum bei Andarig ${ }^{29}$ wahr zu machen: „Nergal, der Herrscher von Hubšalum, ist bei der Niederlage (der Feinde) Dir und Deinem Heer zur Seite gestanden. Lasse alles, was Du gelobt hast, und ein großes Schwert aus Bronze herstellen, und man möge es dem Nergal, dem Herrscher von Hubšalum, darbringen!“30 Von einem Bronzeschwert (GÍR ZABAR) für Nergal von Hubšalum ist auch in einem anderen, leider noch unpublizierten Brief an Zimrī-Lìm ${ }^{31}$ die Rede; es handelt sich um dieselbe Weihung, die in der Zwischenzeit hergestellt wurde und bei der es nun Transportprobleme gibt. Das Nergal-Heiligtum von Hubšalum ist auch sonst in den Briefen an Zimrī-Lìm ${ }^{32}$ und einem altassyrischen Vertrag aus Tell Leilan ${ }^{33}$ belegt, und der Ort muß unweit der Stadt Andarig im Gebiet südlich des Jebel Sinjar gesucht werden. ${ }^{34}$

Auch ein hethitischer König weihte nach einem Sieg ein mit einer Inschrift versehenes Schwert. Das Stück kam 1991 bei Straßenbauarbeiten im Gebiet von Huattuša (Boğazköy) zutage; 35 erhalten ist nur die $79 \mathrm{~cm}$

${ }^{29} \mathrm{Zu}$ diesem Feldzug s. D. Charpin, Prophètes et rois dans le Proche-Orient amorrite. Nouvelles données, nouvelles perspectives, in: D. Charpin/J.-M. Durand (Hg.), Recueil d'études à la mémoire d'André Parrot. Florilegium Marianum 6 (Paris 2002) 30, und D. Charpin/N. Ziegler, Mari et le Proche-Orient à l'époque amorrite. Florilegium Marianum 5 (Paris 2003) 234f.

30 A. $4260=$ ARMT 26/1 194: 24-31: dNè-iri ${ }_{11}$-[ga]l [LU]GAL Hu-ub-ša-limki i-na da$a m_{7}$-de-e-em a-na [i-d] i-ka ù i-di um-ma-na-ti-ka iz-[zi]-iz ma-al ta-ak-ru-bu ù nam-șara-am ZABAR GAL $a$-n $a$ d Nè-iri ${ }_{11}$-gal [L]UGAL Hu-ub-sa-limki $l i-i b$-lu. Für den Hintergrund dieses Briefs s. Charpin in: Recueil d'études (Anm. 29), 29-31.

31 T. 316, s. Charpin in: Recueil d'études (Anm. 29), 30 Anm. 188 und 30f. Anm. 190.

32 A. $2203=$ ARMT 26/2 419 Rs. 3'-21'; M. 13741 = ARMT 27 32: 7 (dort als ${ }^{d} A-m i$ $H u-u b-<\breve{s} a>l_{i m}{ }^{\mathrm{ki}}$, zur Gleichsetzung von Nergal und Amûm s. J.-M. Durand, Le dieu des Enfers à Mari, NABU 1993/60).

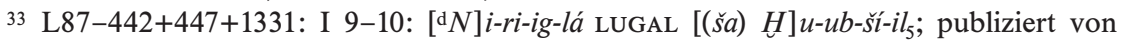
J. Eidem, An Old Assyrian Treaty from Tell Leilan, in: D. Charpin/F. Joannès (Hg.), Marchands, diplomates et empereurs. Études sur la civilisation mésopotamienne offertes à Paul Garelli (Paris 1991) 185-207.

34 Charpin, NABU 1989/76 (aufgrund von A. 2203), vgl. auch Eidem in: Marchands, 205 und Charpin in: Recueil d'études (Anm. 29) 30.

35 Publikation: A. Ertekin/I. Ediz/A. Ünal, The Hittite Sword from Boğazköy-Hattuša, Found in 1991 and Its Akkadian Inscription - 1991'de Boğazköy-Hattusa'da Bulunan Hitit Kılıcı ve Üzerindeki Akadca Adak Yazısı, Müze/Museum 4 (1990-91) 46-52; [A. Ünal,] Ein hethitisches Schwert mit akkadischer Inschrift aus Boğazköy, Antike Welt 23 (1992) 256f.; A. Ünal, Boğazköy Kılıcının Üzerindeki Akadca Adak Yazısı Yeni Gözlemler, in: M. J. Mellink u.a. (Hg.), Aspects of Art and Iconography: Anatolia and Its Neighbors. Studies in Honor of Nimet Özgüç (Ankara 1993) 727-730; A. Ertekin/I. Ediz, The Unique Sword from Boğazköy/Hattuša, in: Aspects of Art, 719-725. Zur Debatte, ob dieses Schwert einer ägäischen oder anatolischen Werkstatt entstammt, s. zuletzt P. Taracha, Is Tuthaliya's Sword really Aegean?, in: G. Beckman 
lange Klinge, entlang der eine akkadische Inschrift in einer Zeile angebracht ist: „Als Tuthalija, der große König, das Land Assuwa vernichtete, hat er diese Schwerter dem Teššub, seinem Herrn, geweiht (wörtl. hinaufgebracht)." ${ }^{36}$ Nachdem dieser Feldzug aus den sogenannten Annalen des Tuthalija ${ }^{37}$ bekannt ist, kann das Schwert Tuthalija I. (15./14. Jh. ${ }^{38}$ ) zugewiesen werden. ${ }^{39}$

Ein weiterer Beleg für eine Waffenweihung nach einer erfolgreich geschlagenen Schlacht ist einer Annaleninschrift Tiglatpilesers III. (744-727) aus seinem Palast in Kalhu (Nimrud) zu entnehmen, und - anders als die Zeugnisse für entsprechende Stiftungen im Heiligtum von Hubšalum und für den anatolischen Wettergott - führt uns diese Quelle auch in jenes Gebiet, aus dem die Waffen mit den (Weih-)Inschriften der babylonischen Herrscher des späten zweiten Jahrtausends stammen. Der assyrische König weihte nach der Eroberung von Bìt-Ištar, einem Fürstensitz im Zagros, im Jahr 737 im örtlichen Tempel, den wir aufgrund weiterer Textquellen als ein Heiligtum der Ištar identifizieren können, ${ }^{40}$ eine mit einer Inschrift versehene Waffe: „Damals fertigte ich einen spitzen Pfeil aus Eisen. Ich schrieb die Leistungen Aššurs, meines Herrn, darauf und montierte (ihn) vor dem Quellteich von Bït-Ištar.“41 Bït-Ištar kann möglicherweise mit Ravānsar, 57 km nordwestlich von Kermanshah, identifiziert werden, da es an diesem Ort einen kleinen See gibt, der von drei Quellen gespeist wird; seine geographische Position läßt sich mit den Informationen über Bït-Ištar aus den assyrischen Inschriften gut vereinbaren. ${ }^{42}$

u.a. (Hg.), Hittite Studies in Honor of Harry H. Hoffner Jr. (Winona Lake 2003) 367-376 (mit früherer Literatur).

36 i-nu-ma ${ }^{1} D u$-ut-ḩa-li-ia LUGAL GAL KUR.URU. $A$-aš-šu-wa ú-hal-liq GÍR.HII.A an-nu-tim $a-n a{ }^{\mathrm{d} I M}$ be-lí-šu ú-še-li.

37 CTH 142, s. H. Klengel, Geschichte des hethitischen Reiches. HdO 34 (Leiden 1998) 104f., und T. Bryce, The Kingdom of the Hittites (Oxford 2005) 124-127.

38 Zum Problem der absoluten Datierung der Regierungszeiten der hethitischen Könige s. zuletzt G. Wilhelm, Generation Count in Hittite Chronology, in: H. Hunger/R. Pruszinzsky (Hg.), Mesopotamian Dark Age Revisited (Wien 2004) 71-77.

${ }^{39}$ Klengel, Geschichte, 104. Zu beachten ist dabei jedoch, daß die Zuweisung der Annalen an Tuthalija I. zwar weitgehend Konsens der modernen Forschung ist, aber dennoch nicht völlig gesichert ist, s. dazu Klengel a. O. 105 mit Anm. 84.

40 K. Radner, A Median Sanctuary at Bït-Ištar, in: G. B. Lanfranchi u.a. (Hg.), Continuity of Empire (?): Assyria, Media, Persia. History of the Ancient Near East Monographs 5 (Padua 2003) 121-123, 128.

${ }^{41} \mathrm{H}$. Tadmor, The Inscriptions of Tiglath-Pileser III, King of Assyria (Jerusalem 1994) 70: Kalhu Annals 14*: 8-9 [= Kalhu Annals 4: 6-7]: ina U U$_{4}$-me-š́u-ma mul-mul-lu AN.BAR zaq-tú DÙ-uš li-ta-at Ǎs-šur EN-ia i-na UGU-hi ášsțur ina UGU nam-ba- $i$ ša URU.É-d15 ú-kin; s. dazu Radner in: Continuity, 121.

42 Radner in: Continuity, 127. 
Die Stadt Bït-Ištar ist aufgrund ihres akkadischen Namens und wegen der Tatsache, daß Tiglatpileser überhaupt Angaben zur kultischen Bedeutung des Ortes macht, unter den Siedlungen im Zagrosgebirge mit „Til-Aššurî, das in der Sprache der Leute von Mihnrānu Pitānu heißt,“43 zu vergleichen. Auch hier nahm Tiglatpileser III. nach Ausweis seiner Inschriften im Jahr 737 am Kultgeschehen teil, indem er am örtlichen Marduk-Heiligtum Opfer durchführen ließ.44 Von diesen beiden Belegen abgesehen, fehlt in den assyrischen Quellen jedwede Angabe über lokale Kulte und Gottheiten im iranischen Hochland, und aufgrund der Tatsache, daß beide Orte akkadische Namen (Bït-Ištar = „Haus der Ištar“; Til-Aššurî = „Tell der Assyrer") tragen und in ihren Tempeln Götter verehrt wurden, die zumindest die Assyrer mit mesopotamischen Namen belegten, liegt es nahe, in Bìt-Ištar und Til-Aššurî, ebenso wie in „Silhazi, das man ,Festung der Babylonier" nennt" ${ }^{45}$, Siedlungen zu vermuten, die auf alte Verbindungen zum Zweistromland zurückblicken konnten, und aufgrund der historischen Gegebenheiten können wir sicherlich annehmen, daß diese Beziehungen auch in der Zeit der Kassiten und der nachfolgenden Dynastien bestanden. ${ }^{46}$

Damit aber können wir im Zagrosgebiet seit dem zweiten vorchristlichen Jahrtausend Heiligtümer belegen, die in Städten mit engen Verbindungen zu Mesopotamien bestanden und in denen Gottheiten verehrt wurden, die (auch) unter mesopotamischen Namen bekannt waren. Anstatt anzunehmen, daß die „Luristanbronzen“ mit Inschriften babylonischer Herrscher und Würdenträger als Beutestücke aus Tempeln in der mesopotamischen Tiefebene ins iranische Hochland verschleppt wurden, ist es weitaus plausibler zu postulieren, daß die Babylonier Weihgaben in den Zagros-Heiligtümern stifteten und damit einer Tradition folgten, die Tiglatpileser III. Jahrhunderte später fortsetzte. Für die beiden Äxte Nebukadnedzars I. und den Dolch des Eriba-Nusku, die jeweils dem Gott Marduk geweiht sind, können wir sogar vielleicht konkret im Marduk-Schrein von Til-Aššurî den von ihren Stiftern intendierten Aufbewahrungsort vermuten.

43 R. Borger, Die Inschriften Asarhaddons, Königs von Assyrien, AfO-Bh. 9 (Graz 1956) 51: Episode 10: 57-58.

44 Tadmor, Inscriptions, 72: Kalhu Annals 15:12: UdU.SISKUR.MEŠ KÙ.MEŠ $a-n a{ }^{\mathrm{d}} \mathrm{A}-$ MAR.UTU $a$-šib KUR.DUL- $\bar{A} \check{s}$-šu-ri $a q-q i$; dazu Radner in: Continuity, 121.

45 Tadmor, Inscriptions, 72: Kalhu Annals 15:11: KUR.Si-il-ha-zi ša dan-nu-tú ša DUMU KÁ.DINGIR.RA ${ }^{\mathrm{ki}} i-q a b-b u-\check{s} u-u ́-n i$.

46 s. dazu K. Radner, An Assyrian View on the Medes, in: G. B. Lanfranchi u.a. (Hg.), Continuity (Anm. 40) 49. 